\title{
ON COMPLETE CONVERGENCE IN A BANACH SPACE
}

\section{ANNA KUCZMASZEWSKA}

Technical University

ul. Bernardyńska 13

20-109 Lublin, Poland

DOMINIK SZYNAL

Institute of Mathematics, UMCS

Plac Marii Curie-Skłodowskiej 1

20-0.31 Lublin, Poland

(Received May 19, 1992 and in revised form January 1, 1993)

ABSTRACT: Sufficient conditions are given under which a sequence of independent random elements taking values in a Banach space satisfy the Hsu and Robbins law of large numbers. The complete convergence of random indexed sums of random elements is also considered.

KEY WORDS AND PHRASES : complete convergence, strong law of large numbers, random elements, Banach space, random indexed sums.

1991 AMS SUBJECT CLASSIFICATION CODES. 60F15, 60B12.

\section{INTRODUCTION}

Let $\left\{X_{n}, n \geq 1\right\}$ be a sequence of independent random elements taking values in a separable Banach space $(B,\|\|)$. Put $S_{n}=\sum_{i=1}^{n} X_{i}$. A sequence $\left\{X_{n}, n \geq 1\right\}$ of random elements is said to satisfy the law of large numbers of Hsu-Robbins type if for any given $\varepsilon>0$

$$
\sum_{n=1}^{\infty} P\left[\left\|S_{n}\right\| \geq \varepsilon n\right]<\infty .
$$

Hsu and Robbins [1] proved that the existence of the second moment of independent, identically distributed random variables for which $E X_{1}=0$, implies the Hsu-Robbins type law of large numbers. Erdös [2] showed that the existence of the second moment of independent, identically distributed random variables and the condition $E X_{1}=0$ is also the necessary one for the Hsu-Robbins type law of large numbers. Considerations concerning (1.1) for sequences and subsequences of independent, identically distributed random variables can be found in Katz [3], Baum, Katz [4], Asmussen, Kurtz [5] and Gut [6]. The results in those cases are given under the assumption when there exists a finite moment of order $r(1<r \leq 2)$.

Some conditions, which guarantee the convergence of (1.1) for sequences and subsequences in the case nonidentically distributed random variables can be found in Duncan, Szynal [7], Bartoszyński, Puri [8] and Kuczmaszewska, Szynal [9], [10]. For instance, it has been shown in Duncan, Szynal [7] that if a sequence $\left\{X_{n} . n \geq 1\right\}$ of independent random variables with $E X_{n}=0$ and 
E. $X_{n}^{2}<x . n \geq 1$ satisli the conditions

$$
\sum_{n=1}^{\infty} \sum_{i=1}^{n} P[|\mathcal{X},| \geq n \Xi]<\infty .
$$

$$
\sum_{n=1}^{n} n^{-4} \sum_{i=1}^{n} E\left(X_{i} I\left[\left|X_{i}\right|<n \xi\right]-E X_{i} I\left[\left|X_{i}\right|<n \varepsilon\right]\right)^{4}<\infty
$$

$$
\sum_{n=1}^{\infty} n^{-1} \sum_{m=2}^{n} \sigma^{2}\left(X_{m} I\left[\left|X_{m}\right|<n \varepsilon\right]\right) \sum_{i=1}^{m-1} \sigma^{2}\left(X_{t} I\left[\left|X_{i}\right|<n \varepsilon\right]\right)<\infty
$$

$$
\sum_{n=1}^{\infty} n^{-4}\left(\sum_{i=1}^{n} E\left(X_{i} I\left[\left|X_{i}\right|<n \varepsilon\right]\right)\right)^{4}<\infty
$$

then

$$
\sum_{n=1}^{\infty} P\left[\left|S_{n}\right| \geq n \varepsilon\right]<\infty
$$

The following example shows that the assumptions (i)-(iv) which are sufficient conditions for (1.1) in the case of independent random variables are not sufficient if we consider sequences of independent random elements taking values in Banach space $\mathrm{B}$.

EXAMPLE. Let $l^{1}$ denote the separable Banach space

$$
l^{1}=\left\{x \in R^{\infty},\|x\|=\sum_{n=1}^{\infty}\left|x_{n}\right|<\infty\right\}
$$

and $e^{n}$ denote the element having 1 for its $\mathrm{n}$-th coordinate and 0 in the other coordinates.

Let $\left\{\xi_{n}, n \geq 1\right\}$ be a sequence of independent random variables defined as follows $P\left[\xi_{n}=1\right]=$ $P\left[\xi_{n}=-1\right]=1 / 2, n \geq 1$, and define $X_{n}=\xi_{n} e^{n}, n \geq 1$. Thus $\left\{X_{n}, n \geq 1\right\}$ is a sequence of independent $l^{1}$-valued random elements with symmetric distributions, such that $E X_{n}=0$, $E\left\|X_{n}\right\|^{2}=1, E\left\|X_{n}\right\|^{4}=1, n \geq 1$, and $\left\{X_{n}, n \geq 1\right\}$ satisfies the assumptions (i)-(iv) but $\left\|n^{-1} \sum_{i=1}^{n} X_{i}\right\|=n^{-1} \sum_{i=1}^{n} 1=1$, which shows that the condition $\sum_{n=1}^{\infty} P\left[\left\|S_{n}\right\| \geq n \varepsilon\right]<\infty$ does not hold for all $\varepsilon>0$.

The aim of this note is to give sufficient conditions, which guarantee the Hsu-Robbins type of large numbers for independent random elements taking values in Banach space B.

\section{PRELIMINARIES}

We need now an extension of Hoffman-Jörgensen inequality ( cf. Hoffmann- Jörgensen [11], and Gut [6]).

LEMMA 1. Let $\left\{X_{n}, n \geq 1\right\}$ be a sequence of independent random elements taking values in a real separable Banach space $(B,\|\|)$ with a symmetric distribution. Then for every $j=1,2, \ldots, n$ and $t>0$

$$
P\left[\left\|S_{n}\right\| \geq 3^{\jmath} t\right] \leq C_{3} \sum_{i=1}^{n} P\left[\left\|X_{\imath}\right\| \geq t\right]+D_{\jmath}\left(P\left[\left\|S_{n}\right\| \geq t\right]\right)^{2^{\jmath}},
$$

where $C_{\jmath}$ and $D_{\jmath}$ are positive constants depending only on j. 
PROOF. LeN $T=m, \int\left\{n \geq 1,\left\|S_{n}\right\| \geq t\right\}$. Then

$$
\begin{gathered}
P\left[\left\|S_{n}\right\| \geq 3 t\right]=\sum_{i=1}^{n} P\left[\left\|S_{n}\right\| \geq 3 t, T=\imath\right] \\
=\sum_{i=1}^{n} P\left[\left\|S_{n}\right\| \geq 3 t,\left\|S_{1}\right\|<t, \ldots,\left\|S_{i-1}\right\|<t,\left\|S_{i}\right\| \geq t\right] \\
=\sum_{i=1}^{n} P\left[\left\|S_{n}-S_{i}+S_{i-1}+X_{i}\right\| \geq 3 t,\left\|S_{1}\right\|<t, \ldots,\left\|S_{i-1}\right\|<t,\left\|S_{i}\right\| \geq t\right] \\
\leq \sum_{i=1}^{n} P\left[\left\|S_{n}-S_{i}\right\| \geq 3 t-\left\|S_{i-1}\right\|-\left\|X_{i}\right\|,\left\|S_{1}\right\|<t, \ldots,\left\|S_{i-1}\right\|<t,\left\|S_{i}\right\| \geq t\right] \\
\leq \sum_{i=1}^{n} P\left[\left\|S_{n}-S_{i}\right\| \geq 2 t-\left\|X_{i}\right\|, T=i\right] \leq \sum_{i=1}^{n} P\left[\left\|X_{i}\right\| \geq t, T=i\right] \\
+\sum_{i=1}^{n} P\left[\left\|S_{n}-S_{i}\right\| \geq t, T=i\right] \leq \sum_{i=1}^{n} P\left[\left\|X_{i}\right\| \geq t\right] \\
+\sum_{i=1}^{n} P\left[\left\|S_{n}-S_{i}\right\| \geq t\right] \cdot P[T=i] .
\end{gathered}
$$

Moreover,

$$
\begin{gathered}
P\left[\left\|S_{n}-S_{\imath}\right\| \geq t\right] \leq P\left[\max \left(\left\|S_{n}-S_{\imath}\right\|,\left\|S_{n}-S_{\imath}+S_{\imath}\right\|\right) \geq t\right] \\
\leq 2 P\left[\left\|S_{n}\right\| \geq t\right],
\end{gathered}
$$

as $S_{n}-S_{\imath}$ and $S_{\imath}$ are independent, symmetrically distributed random elements.

Hence

$$
\begin{gathered}
P\left[\left\|S_{n}\right\| \geq 3 t\right] \leq \sum_{i=1}^{n} P\left[\left\|X_{\imath}\right\| \geq t\right]+2 P\left[\left\|S_{n}\right\| \geq t\right] \cdot \sum_{i=1}^{n} P[T=i] \\
\leq \sum_{i=1}^{n} P\left[\left\|X_{\imath}\right\| \geq t\right]+2 P\left[\left\|S_{n}\right\| \geq t\right] \cdot P\left[\max _{i \leq j \leq n}\left\|S_{j}\right\| \geq t\right] \\
\leq \sum_{i=1}^{n} P\left[\left\|X_{\imath}\right\| \geq t\right]+4\left(P\left[\left\|S_{n}\right\| \geq t\right]\right)^{2} .
\end{gathered}
$$

By the induction principle, we get

$$
\begin{gathered}
P\left[\left\|S_{n}\right\| \geq 3^{\jmath+1} t\right]=P\left[\left\|S_{n}\right\| \geq 3 \cdot 3^{\jmath} t\right] \\
\leq \sum_{i=1}^{n} P\left[\left\|X_{\imath}\right\| \geq 3^{\jmath} t\right]+4\left(P\left[\left\|S_{n}\right\| \geq 3^{\jmath} t\right]\right)^{2} \\
\leq \sum_{i=1}^{n} P\left[\left\|X_{\imath}\right\| \geq t\right]+4\left(C_{\jmath} \sum_{i=1}^{n} P\left[\left\|X_{\imath}\right\| \geq t\right]+D_{\jmath} \cdot P^{2^{\jmath}}\left[\left\|S_{n}\right\| \geq t\right]\right)^{2} \\
\leq C_{J+1} \sum_{i=1}^{n} P\left[\left\|X_{\imath}\right\| \geq t\right]+D_{\jmath+1}\left(P\left[\left\|S_{n}\right\| \geq t\right]\right)^{2^{\jmath+1}} .
\end{gathered}
$$

Moreover, we shall use the following lemmas. 
I.EXIMA 2. ( Vuinki $[1 \cdot 2]$ ) Let $x_{1}, \ldots . X_{n}$ be independent B-ralued random elements with $l:\left\|X_{i}\right\|<\infty(1=1 \ldots, n)$. I, et $\mathcal{F}_{h}$ be the $\sigma$-ficld generated by $\left(X_{1} \ldots \ldots X_{h}\right),(k=1, \ldots, n)$ and lot $\mathcal{F}_{0}=\{\emptyset, \Omega\}$. 'Then for $1 \leq k \leq n$

$$
\left|E\left(\left\|\varphi_{n}\right\| \mid \mathcal{F}_{h}\right)-\ell\left(\left\|, \varphi_{n}\right\| \mid \mathcal{F}_{h-1}\right)\right| \leq\left\|\cdot \mathcal{X}_{h}\right\|+E\left\|X_{h}\right\|
$$

LFMIIA 3. ( Locve [1:3] ) For every $z>0$

$$
\begin{aligned}
& P\left[\left\|X^{\cdot}-\operatorname{med} X^{-}\right\| \geq \Xi\right] \leq 2 \cdot P\left[\left\|\cdot X^{-s}\right\| \geq \varepsilon\right] \text {. } \\
& P\left[\sup _{1 \leq n}\left\|X_{j}-\operatorname{med} X_{1}\right\| \geq \varepsilon\right] \leq 2 \cdot P\left[\underset{j \leq n}{\sup }\left\|X_{j}^{s}\right\| \geq \varepsilon\right] .
\end{aligned}
$$

where $A^{s}$ is a symmetrized ression of $X$.

In what follows we shall use the strong law of large numbers for a sequence of independent, identically distributed random elements $\left\{X_{n}, n \geq 1\right\}$ in a separable Banach space given in Taylor $[14]$.

THEOREM. Let $\left\{X_{n}, n \geq 1\right\}$ be a sequence of independent identically distributed B-valued random elements such that $E\left\|X_{1}\right\|<\infty$.

Then $\left\|n^{-1} \sum_{i=1}^{n} X_{i}-E . X_{1}\right\| \rightarrow 0$ a.s. as $n \rightarrow \infty$.

\section{RESULTS}

THEOREM 1. Let $\left\{X_{n}, n \geq 1\right\}$ be a sequence of independent, symmetrically distributed, B-valued random elements. Suppose that $\left\{n_{h}, k \geq 1\right\}$ is a strictly increasing sequence of positive integers. If for some positive integer $\mathrm{j}$ and any given $\varepsilon>0$

$$
\sum_{k=1}^{\infty}\left(n_{k}^{-4} \sum_{i=1}^{n_{h}} E\left\|X_{i}\right\|^{4} I\left[\left\|X_{i}\right\|<n_{k} \varepsilon\right]\right)^{2^{\jmath}}<\infty
$$

$$
\sum_{k=1}^{\infty}\left(n_{k}^{-4} \sum_{m=2}^{n_{k}} E\left\|X_{m}\right\|^{2} I\left[\left\|X_{m}\right\|<n_{k} \varepsilon\right] \sum_{i=1}^{m-1} E\left\|X_{\imath}\right\|^{2} I\left[\left\|X_{\imath}\right\|<n_{k} \varepsilon\right]\right)^{2^{\jmath}}<\infty,
$$

then

$$
\sum_{k=1}^{\infty} P\left[\left\|S_{n_{k}}\right\| \geq n_{k} \varepsilon\right]<\infty
$$

iff

$$
\left\|S_{n_{k}} / n_{k}\right\| \rightarrow 0 \text { in probability as } k \rightarrow \infty
$$

PROOF. It is enough to show that under the conditions (i)-(iv) $\left\|S_{n_{k}} / n_{k}\right\| \rightarrow 0$ in probability as $k \rightarrow \infty$ implies that $\sum_{k=1}^{\infty} P\left[\left\|S_{n_{k}}\right\| \geq n_{k} \varepsilon\right]<\infty$.

Put $X_{\jmath}^{\prime}=X_{\jmath} I\left[\left\|X_{\jmath}\right\|<n_{k} \varepsilon\right] . S_{n}^{\prime}=\sum_{i=1}^{n} X_{\imath}^{\prime}$ and $Y_{n_{k}, 2}=E\left(\left\|S_{n_{k}}^{\prime}\right\| \mid \mathcal{F}_{\imath}\right)-E\left(\left\|S_{n-k}^{\prime}\right\| \mid \mathcal{F}_{t-1}\right)$ where $\mathcal{F}_{\imath}=\sigma\left(X_{1}^{\prime}, X_{2}^{\prime}, \ldots, X_{\imath}^{\prime}\right)$ and $\mathcal{F}_{0}=\{\emptyset, \Omega\}$. Then we have 
$P\left[\left\|S_{n_{k}}\right\| \geq n_{k} \xi\right] \leq\left(^{\prime}, \sum_{i=1}^{n_{k}} P\left[\left\|X_{i}\right\| \geq n_{k} \varepsilon / 3^{\jmath}\right]+D_{\jmath}\left(P\left[\left\|S_{n_{\alpha}}\right\| \geq n_{k} \varepsilon / 3^{\jmath}\right]\right)^{2^{\jmath}}\right.$.

Moreover,

$$
\begin{gathered}
\sum_{k=1}^{\infty}\left(P\left[\left\|S_{n_{A}}\right\| \geq n_{k} \varepsilon / 3^{\jmath}\right]\right)^{2^{\jmath}} \\
\leq 2^{2 \jmath-1}\left\{\sum_{k=1}^{\infty}\left(\sum_{i=1}^{n_{k}} P\left[\left\|X_{i}\right\| \geq n_{k} \varepsilon / 3^{\jmath}\right]\right)^{2^{\jmath}}+\sum_{k=1}^{\infty}\left(P\left[\left\|S_{n_{k}}^{\prime}\right\| \geq n_{k} \varepsilon / 3^{\jmath}\right]\right)^{2^{\jmath}}\right\} .
\end{gathered}
$$

Note that $\left\|S_{n_{k}}^{\prime}\right\|-E\left\|S_{n_{k}}^{\prime}\right\|=\sum_{i=1}^{n_{k}} Y_{n_{k}, 1}$ and

$$
\begin{gathered}
\sum_{k=1}^{\infty}\left(P\left[\mid\left\|S_{n_{k}}^{\prime}\right\|-E\left\|S_{n_{k}}^{\prime}\right\| I \geq n_{k} \varepsilon / 3^{\jmath}\right]\right)^{2^{\jmath}}=\sum_{k=1}^{\infty}\left(P\left[\left(\sum_{i=1}^{n_{k}} Y_{n_{k}, 1^{\prime}}\right)^{2} \geq n_{k}^{2}\left(\varepsilon / 3^{\jmath}\right)^{2}\right]\right)^{2 \jmath} \\
=\sum_{k=1}^{\infty}\left\{P\left[\sum_{i=1}^{n_{k}} Y_{n_{k}, t}^{2}+2 \sum_{m=2}^{n_{k}} Y_{n_{k}, m} \sum_{i=1}^{m-1} Y_{n_{k}, t} \geq n_{k}^{2}\left(\varepsilon / 3^{\jmath}\right)^{2}\right]\right\}^{2^{\jmath}} \\
\leq \sum_{k=1}^{\infty}\left(P\left[\sum_{i=1}^{n_{k}} Y_{n_{k}, t}^{2} \geq n_{k}^{2}\left(\varepsilon / 3^{\jmath}\right)^{2} / 2\right]+P\left[\sum_{m=2}^{n_{k}} Y_{n_{k}, m} \sum_{i=1}^{m-1} Y_{n_{k}, t} \geq n_{k}^{2}\left(\varepsilon / 3^{J}\right)^{2} / 4\right]\right)^{2} .
\end{gathered}
$$

Now putting $Z_{n_{k}, t}=Y_{n_{k}, \mathrm{l}}^{2}-E Y_{n_{k}, \mathrm{l}}^{2}$ and using the inequality (2.2) we get for $\varepsilon^{\prime}=\left(\varepsilon / 3^{3}\right)^{2} / 2$

$$
\begin{aligned}
& \sum_{k=1}^{\infty}\left(P\left[\left|\sum_{i=1}^{n_{k}} Z_{n_{k}, 2}\right| \geq n_{k}^{2} \varepsilon^{\prime}\right]\right)^{2^{\jmath}} \leq\left(\varepsilon^{\prime}\right)^{-2^{j+1}} \sum_{k=1}^{\infty}\left(n_{k}^{-4} E\left|\sum_{i=1}^{n_{k}} Z_{n_{k}, l^{2}}\right|^{2}\right)^{2^{\jmath}}
\end{aligned}
$$

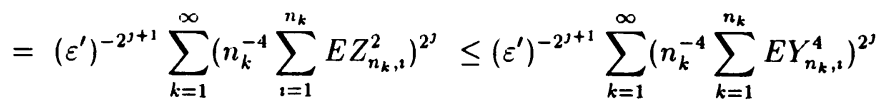

$$
\begin{aligned}
& \leq\left(\varepsilon^{\prime}\right)^{-2^{3+1}} \cdot 2^{2^{3+2}} \sum_{k=1}^{\infty}\left(n_{k}^{-4} \sum_{i=1}^{n_{k}} E\left\|X_{i}^{\prime}\right\|^{4}\right)^{2^{\jmath}}<\infty .
\end{aligned}
$$

Moreover, we see that (ii) and (iii) imply

$$
n_{k}^{-2} \sum_{i=1}^{n_{k}} E Y_{n_{k}, t}^{2} \leq 8 n_{k}^{-2} \sum_{i=1}^{n_{k}} E\left\|X_{i}^{\prime}\right\|^{2}=o(1)
$$

as

$$
\left(n_{k}^{-2} \sum_{i=1}^{n_{k}} E\left\|X_{\imath}^{\prime}\right\|^{2}\right)^{2}=n_{k}^{-4} \sum_{i=1}^{n_{k}} E\left\|X_{i}^{\prime}\right\|^{4}+n_{k}^{-4} 2 \sum_{m=2}^{n_{k}} E\left\|X_{m}^{\prime}\right\|^{2} \sum_{i=1}^{m-1} E\left\|X_{i}^{\prime}\right\|^{2}
$$

implies

$$
\left(n_{k}^{-2} \sum_{i=1}^{n_{k}} E\left\|X_{\imath}^{\prime}\right\|^{2}\right)^{2} \rightarrow 0 \text { as } k \rightarrow \infty .
$$

Now we see that $\left\{Y_{n_{k}, i} \sum_{j=1}^{i-1} Y_{n_{k}, j}, 2 \leq i \leq n\right\}$ and $\left\{Y_{n_{k}, r}, 1 \leq i \leq n\right\}$ are martingale differences for fixed $\mathrm{n}$. Therefore 


$$
\begin{aligned}
& \sum_{k=1}^{\infty}\left(P\left[\sum_{m=2}^{n_{k}} Y_{n_{k}, m} \sum_{i=1}^{m-1} Y_{n_{k}, t} \geq n_{k}^{2} \varepsilon^{\prime} / 2\right]\right)^{2} \\
& \leq 2^{2^{j+1}}\left(\varepsilon^{\prime}\right)^{-2^{j+1}} \sum_{k=1}^{\infty}\left\{n_{k}^{-4} \sum_{m=2}^{n_{k}} E\left(Y_{n_{k}, m} \sum_{i=1}^{m-1} Y_{n_{k}, 2}\right)^{2}\right\}^{2 j} \\
& \leq 2^{2^{\prime+1}}\left(\varepsilon^{\prime}\right)^{-2^{\prime+1}} \sum_{k=1}^{\infty}\left\{n_{k}^{-4} \sum_{m=2}^{n_{k}} E\left[\left(\left\|X_{m}^{\prime}\right\|+E\left\|X_{m}^{\prime}\right\|\right)^{2}\left(\sum_{i=1}^{m-1} Y_{n_{k}, 2}\right)^{2}\right]\right\}^{2 \prime} \\
& \leq 2^{2^{j+1}}\left(\varepsilon^{\prime}\right)^{-2^{3+1}} \sum_{k=1}^{\infty}\left\{n_{k}^{-4} \sum_{m=2}^{n_{k}} E\left(\left\|X_{m}^{\prime}\right\|+E\left\|X_{m}^{\prime}\right\|\right)^{2} \sum_{i=1}^{m-1} E\left(\left\|X_{i}^{\prime}\right\|+E\left\|X_{i}^{\prime}\right\|\right)^{2}\right\}^{2^{\prime}} \\
& \leq A_{J} \sum_{k=1}^{\infty}\left\{n_{k}^{-4} \sum_{m=2}^{n_{k}} E\left\|X_{m}^{\prime}\right\|^{2} \sum_{i=1}^{m-1} E\left\|X_{\imath}^{\prime}\right\|^{2}\right\}^{2 \prime}<\infty,
\end{aligned}
$$

where $A_{\jmath}$ is a positive constant depending only on $\mathrm{j}$ and $\varepsilon$.

Thus we have proved that

$$
\left.\sum_{k=1}^{\infty}\left(P\left[\mid\left\|S_{n_{k}}^{\prime}\right\|-E\left\|S_{n_{k}}^{\prime}\right\|\right] \geq n_{k} \varepsilon / 3^{\jmath}\right]\right)^{2^{\jmath}}<\infty
$$

which implies that

$$
P\left[\left\|S_{n_{k}}^{\prime}\right\|-E\left\|S_{n_{k}}^{\prime}\right\| \mid \geq n_{k} \varepsilon\right] \rightarrow 0 \text { as } k \rightarrow \infty .
$$

Moreover, we state that (3.1) and (i) imply

$$
\begin{gathered}
P\left[\left\|S_{n_{k}}^{\prime}\right\| \geq n_{k} \varepsilon\right]=P\left[\left\|S_{n_{k}}^{\prime}\right\| \geq n_{k} \varepsilon, S_{n_{k}}=S_{n_{k}}^{\prime}\right]+P\left[\left\|S_{n_{k}}^{\prime}\right\| \geq n_{k} \varepsilon, S_{n_{k}} \neq S_{n_{k}}^{\prime}\right] \\
\leq P\left[\left\|S_{n_{k}}\right\| \geq n_{k} \varepsilon\right]+\sum_{i=1}^{n_{k}} P\left[\left\|X_{\imath}\right\| \geq n_{k} \varepsilon\right] \rightarrow 0 \text { as } k \rightarrow \infty
\end{gathered}
$$

or

$$
P\left[\left\|S_{n_{k}}^{\prime}\right\| \geq n_{k} \varepsilon\right] \rightarrow 0 \text { as } k \rightarrow \infty .
$$

Hence by (3.3) and (3.4) we get

$$
E\left\|S_{n_{k}}^{\prime}\right\| / n_{k} \rightarrow 0 \text { as } k \rightarrow \infty
$$

which together with (3.2) gives

$$
\sum_{k=1}^{\infty}\left(P\left[\left\|S_{n_{k}}^{\prime}\right\| \geq n_{k} \varepsilon / 3^{\jmath}\right]\right)^{2^{\jmath}}<\infty
$$

Taking into account that

$$
\begin{gathered}
\sum_{k=1}^{\infty}\left(P\left[\left\|S_{n_{k}}\right\| \geq n_{k} \varepsilon / 3^{\jmath}\right]\right)^{2^{\jmath}} \\
\leq 2^{2^{\jmath}-1}\left\{\sum_{k=1}^{\infty}\left(\sum_{i=1}^{n_{k}} P\left[\left\|X_{\imath}\right\| \geq n_{k} \varepsilon / 3^{\jmath}\right]\right)^{2^{\jmath}}+\sum_{k=1}^{\infty}\left(P\left[\left\|S_{n_{k}}^{\prime}\right\| \geq n_{k} \varepsilon / 3^{\jmath}\right]\right)^{2^{\jmath}}\right\}
\end{gathered}
$$

and using (i) we complete the proof of Theorem 1.

COROLLARY 1. Let $\left\{X_{n}, n \geq 1\right\}$ be a sequence of independent, symmetrically distributed, B-valued random elements. Suppose that $\left\{n_{k}, k \geq 1\right\}$ is a strictly increasing sequence of positive integers. If for some positive integer $\mathrm{j}$ and any given $\varepsilon>0$ 


$$
\begin{gathered}
\sum_{k=1}^{\lambda} \sum_{i=1}^{n_{k}} P\left[\left\|\mathcal{X}_{i}\right\| \geq n_{k} \varepsilon / 3^{\jmath}\right]<\infty, \\
\sum_{k=1}^{\lambda}\left(n_{k}^{-2} \sum_{i=1}^{n_{h}} E\left\|X_{i}\right\|^{2} I\left[\left\|X_{i}\right\|<n_{k} \varepsilon\right]\right)^{2^{\jmath}}<\infty,
\end{gathered}
$$

then

$$
\sum_{k=1}^{\infty} P\left[\left\|S_{n_{h}}\right\| \geq n_{h} \varepsilon\right]<\infty
$$

iff

$$
\left\|S_{n_{k}} / n_{k}\right\| \rightarrow 0 \text { in probability as } k \rightarrow \infty .
$$

Now we consider the Hsu and Robbins law of large numbers for subsequences of independent, nonsymmetrically distributed random elements taking values in a real separable Banach space.

THEOREM 2. Let $\left\{X_{n}, n \geq 1\right\}$ be a sequence of independent, B-valued random elements. Suppose that $\left\{n_{h}, k \geq 1\right\}$ is a strictly increasing sequence of positive integers. If for some positive integer $j$ and any given $\varepsilon>0$

$$
\begin{gathered}
\sum_{k=1}^{\infty} \sum_{i=1}^{n_{k}} P\left[\left\|X_{\imath}\right\| \geq n_{k} \varepsilon /\left(2 \cdot 3^{\jmath}\right)\right]<\infty, \\
\sum_{k=1}^{\infty}\left(n_{k}^{-4} \sum_{i=1}^{n_{k}} E\left\|X_{\imath}\right\|^{4} I\left[\left\|X_{\imath}\right\|<2 n_{k} \varepsilon\right]\right)^{2^{\jmath}}<\infty, \\
\sum_{k=1}^{\infty}\left(n_{k}^{-4} \sum_{m=2}^{n_{k}} E\left\|X_{m}\right\|^{2} I\left[\left\|X_{m}\right\|<2 n_{k} \varepsilon\right] \sum_{i=1}^{m-1} E\left\|X_{\imath}\right\|^{2} I\left[\left\|X_{\imath}\right\|<2 n_{k} \varepsilon\right]\right)^{2^{\jmath}}<\infty,
\end{gathered}
$$

then

iff

$$
\sum_{k=1}^{\infty} P\left[\left\|S_{n_{k}}\right\| \geq n_{k} \varepsilon\right]<\infty
$$

$$
\left\|S_{n_{k}} / n_{k}\right\| \rightarrow 0 \text { in probability as } k \rightarrow \infty
$$

PROOF. Assume that $\left\{X_{n}, n \geq 1\right\}$ is a sequence of symmetrically distributed random elements. Then by Theorem 1 we conclude that conditions (I) - (III) are sufficient for the Hsu and Robbins law of large numbers, i.e.

$$
\sum_{k=1}^{\infty} P\left[\left\|S_{n_{k}}\right\| \geq n_{k} \varepsilon\right]<\infty
$$

To remove the symmetry assumption we argue as follows. Let $\left\{X_{n}^{s}, n \geq 1\right\}$ be a sequence of the symmetrized version of $X$, i.e. $X_{k}^{s}=X_{k}-X_{k}^{*}, k \geq 1$, where $X_{k}$ and $X_{k}^{*}$ are independent and have the same distribution. Then by (I) we get for $\varepsilon^{\prime}=\varepsilon / 3^{\jmath}$

$$
\sum_{k=1}^{\infty} \sum_{i=1}^{n_{k}} P\left[\left\|X_{\imath}^{s}\right\| \geq n_{k} \varepsilon^{\prime}\right]=\sum_{k=1}^{\infty} \sum_{i=1}^{n_{k}} P\left[\left\|X_{\imath}-X_{i}^{*}\right\| \geq n_{k} \varepsilon^{\prime}\right]
$$




$$
\leq 2 \sum_{k=1}^{\infty} \sum_{i=1}^{n_{k}} P\left[\left\|\cdot \dot{X}_{i}\right\| \geq n_{h} \varepsilon^{\prime} / 2\right]<\infty
$$

and by (I) and (II) we have

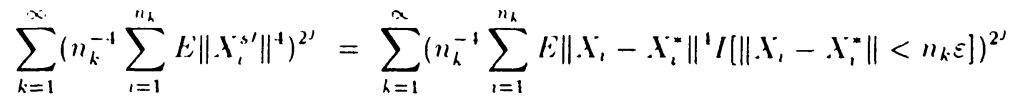

$$
\begin{aligned}
& =\sum_{k=1}^{\lambda}\left(n_{k}^{-1} \sum_{i=1}^{n_{k}} E\left\|X_{i}-X_{i}^{*}\right\|^{4} I\left[\left\|X_{\imath}-X_{i}^{*}\right\|<n_{k} \varepsilon,\left\|X_{i}^{*}\right\|<n_{k} \varepsilon\right]\right. \\
& \left.+n_{k}^{-4} \sum_{i=1}^{n_{k}} E\left\|X_{i}-X_{i}^{*}\right\|^{4} I\left[\left\|X_{i}-X_{i}^{*}\right\|<n_{k} \varepsilon,\left\|X_{i}^{*}\right\| \geq n_{k} \varepsilon\right]\right)^{2} \\
& \leq 2^{2^{3+1}-1} \sum_{k=1}^{\infty}\left(n_{k}^{-1} \sum_{i=1}^{n_{k}} E\left\|X_{i}\right\|^{4} I\left[\left\|X_{i}\right\|<2 n_{k} \varepsilon\right]\right)^{2^{\jmath}}+2^{2^{\jmath}-1} \varepsilon^{2^{\jmath+2}} \sum_{k=1}^{\infty} \sum_{i=1}^{n_{k}}\left(P\left[\left\|X_{i}\right\| \geq n_{k} \varepsilon\right]\right)^{2^{\jmath}}<\infty .
\end{aligned}
$$

Now we see (II) and (III) imply

$$
n_{k}^{-2} \sum_{i=1}^{n_{k}} E\left\|X_{\imath}\right\|^{2} I\left[\left\|X_{\imath}\right\|<2 n_{k} \varepsilon\right] \rightarrow 0 \text { as } k \rightarrow \infty
$$

since

$$
\begin{aligned}
& \left(n_{k}^{-2} \sum_{i=1}^{n_{k}} E\left\|X_{\imath}\right\|^{2} I\left[\left\|X_{\imath}\right\|<2 n_{k} \varepsilon\right]\right)^{2}=n_{k}^{-4} \sum_{i=1}^{n_{k}} E\left\|X_{\imath}\right\|^{4} I\left[\left\|X_{\imath}\right\|<2 n_{k} \varepsilon\right] \\
& +2 n_{k}^{-4} \sum_{m=2}^{n_{k}} E\left\|X_{m}\right\|^{2} I\left[\left\|X_{m}\right\|<2 n_{k} \varepsilon\right] \sum_{i=1}^{m-1} E\left\|X_{i}\right\|^{2} I\left[\left\|X_{i}\right\|<2 n_{k} \varepsilon\right]
\end{aligned}
$$

Therefore by (I), (III) and (3.5) we obtain

$$
\begin{gathered}
\sum_{k=1}^{\infty}\left(n_{k}^{-4} \sum_{m=2}^{n_{k}} E\left\|X_{m}^{s}\right\|^{2} \sum_{i=1}^{m-1} E\left\|X_{\imath}^{s \prime}\right\|^{2}\right)^{2^{\prime}} \\
\leq C\left\{\sum_{k=1}^{\infty}\left(n_{k}^{-4} \sum_{m=2}^{n_{k}} E\left\|X_{m}\right\|^{2} I\left[\left\|X_{m}\right\|<2 n_{k} \varepsilon\right] \cdot \sum_{i=1}^{m-1} E\left\|X_{\imath}\right\|^{2} I\left[\left\|X_{\imath}\right\|<2 n_{k} \varepsilon\right]\right)^{2}\right. \\
+\sum_{k=1}^{\infty}\left(n_{k}^{-2} \sum_{m=2}^{n_{k}} E\left\|X_{m}\right\|^{2} I\left[\left\|X_{m}\right\|<2 n_{k} \varepsilon\right] \cdot \sum_{i=1}^{m-1} P\left[\left\|X_{\imath}\right\| \geq n_{k} \varepsilon\right]\right)^{2^{3}} \\
+\sum_{k=1}^{\infty}\left(n_{k}^{-2} \sum_{m=2}^{n_{k}} P\left[\left\|X_{m}\right\| \geq n_{k} \varepsilon\right] \cdot \sum_{i=1}^{m-1} E\left\|X_{\imath}\right\|^{2} I\left[\left\|X_{\imath}\right\|<2 n_{k} \varepsilon\right]\right)^{2^{\prime}} \\
\left.+\sum_{k=1}^{\infty} \sum_{i=1}^{n_{k}} P\left[\left\|X_{\imath}\right\| \geq n_{k} \varepsilon\right]\right\}<\infty
\end{gathered}
$$

where $\mathrm{C}$ is a positive constant depending only on $\mathrm{j}$ and $\varepsilon$.

Hence by Theorem 1 we obtain

$$
\sum_{k=1}^{\infty} P\left[\left\|S_{n_{k}}^{s}\right\| \geq n_{k} \varepsilon\right]<\infty
$$

Taking into account the symmetrization inequality (2.3)

$$
P\left[\left\|S_{n_{k}} / n_{k}-\operatorname{med}\left(S_{n_{k}} / n_{k}\right)\right\| \geq \varepsilon\right] \leq 2 P\left[\left\|S_{n_{k}}^{s}\right\| \geq n_{k} \varepsilon\right]
$$


we have

$$
\sum_{k=1}^{x} P\left[\left\|. S_{n_{k}} / n_{k}-\operatorname{med}\left(S_{n_{k}} / n_{k}\right)\right\| \geq \varepsilon\right]<\infty
$$

But the assumption $P\left[\left\|\breve{S}_{n_{k}}\right\| \geq n_{k} \varepsilon\right] \rightarrow 0$ as $k \rightarrow \infty$

$$
\left\|\operatorname{med}\left(S_{n_{k}} / n_{k}\right)\right\| \rightarrow 0 \text { as } k \rightarrow \infty \text {, }
$$

which together with

$$
\sum_{h=1}^{\infty} P\left[\left\|S_{n_{k}} / n_{h}-\operatorname{med}\left(S_{n_{k}} / n_{k}\right)\right\| \geq \varepsilon\right]<\infty
$$

gives

$$
\sum_{k=1}^{\infty} P\left[\left\|S_{n_{k}}\right\| \geq n_{k} \varepsilon\right]<\infty
$$

COROLLARY 2. Let $\left\{X_{n}, n \geq 1\right\}$ be a sequence of independent, B-valued random clements. Suppose that $\left\{n_{k}, k \geq 1\right\}$ is a strictly increasing sequence of positive integers. If for some positive integer $\mathrm{j}$ and any given $\varepsilon>0$

$$
\begin{gathered}
\sum_{k=1}^{\infty} \sum_{i=1}^{n_{k}} P\left[\left\|X_{\imath}\right\| \geq n_{k} \varepsilon /\left(2 \cdot 3^{\jmath}\right)\right]<\infty, \\
\sum_{k=1}^{\infty}\left(n_{k}^{-2} \sum_{i=1}^{n_{k}} E\left\|X_{\imath}\right\|^{2} I\left[\left\|X_{\imath}\right\|<2 n_{k} \varepsilon\right]\right)^{2^{\jmath}}<\infty,
\end{gathered}
$$

then

$$
\sum_{k=1}^{\infty} P\left[\left\|S_{n_{k}}\right\| \geq n_{k} \varepsilon\right]<\infty
$$

iff

$$
\left\|S_{n_{k}} / n_{k}\right\| \rightarrow 0 \text { in probability as } k \rightarrow \infty .
$$

COROLLARY 3. Let $\left\{X_{n}, n \geq 1\right\}$ be a sequence of independent, B-valued random elements. Suppose that $\left\{n_{k}, k \geq 1\right\}$ is a strictly increasing sequence of positive integers. If for some positive integer $\mathrm{j}$ and any given $\varepsilon>0$

$$
\begin{gathered}
\sum_{k=1}^{\infty} \sum_{\imath=1}^{n_{k}} P\left[\left\|X_{\imath}\right\| \geq n_{k} \varepsilon /\left(2 \cdot 3^{\jmath}\right)\right]<\infty, \\
\sum_{k=1}^{\infty}\left(n_{k}^{-4} \sum_{i=1}^{n_{k}} E\left\|X_{\imath}\right\|^{4} I\left[\left\|X_{\imath}\right\|<2 n_{k} \varepsilon\right]\right)^{2 \jmath}<\infty, \\
\sum_{k=1}^{\infty}\left(n_{k}^{-2} \sum_{i=1}^{n_{k}} E\left\|X_{\imath}\right\|^{2} I\left[\left\|X_{\imath}\right\|<2 n_{k} \varepsilon\right]\right)^{2^{j+1}}<\infty,
\end{gathered}
$$

then 


$$
\sum_{h=1}^{\infty} P\left[\left\|S_{n_{h}}\right\| \geq n_{h}=\right]<\infty
$$

iff

$$
\left\|S_{n_{k}} / n_{k}\right\| \rightarrow 0 \text { in probability as } k \rightarrow \infty .
$$

Some results concerning the independent identically distributed randon elements can be obtained as corollaries of Theorem 2.

('OROLLARY 4. Let $\left\{X_{n}, n \geq 1\right\}$ be a sequence of independent, identically distributed B-valued random clements. Suppose that $\left\{n_{h}, k \geq 1\right\}$ is a strictly increasing sequence of positive integers. If for some positive integer $\mathrm{j}$ and any given $\varepsilon>0$

$$
\begin{gathered}
\sum_{k=1}^{\infty} n_{k} P\left[\left\|X_{1}\right\| \geq n_{k} \varepsilon /\left(2 \cdot 3^{\jmath}\right)\right]<\infty, \\
\sum_{k=1}^{\infty}\left(n_{k}^{-3} E\left\|X_{1}\right\|^{4} I\left[\left\|X_{1}\right\|<2 n_{k} \varepsilon\right]\right)^{2^{\jmath}}<\infty, \\
\sum_{k=1}^{\infty}\left(n_{k}^{-1} E\left\|X_{1}\right\|^{2} I\left[\left\|X_{1}\right\|<2 n_{k} \varepsilon\right]\right)^{2^{\jmath+1}}<\infty,
\end{gathered}
$$

then

$$
\sum_{k=1}^{\infty} P\left[\left\|S_{n_{k}}\right\| \geq n_{k} \varepsilon\right]<\infty
$$

iff

$$
\left\|S_{n_{k}} / n_{k}\right\| \rightarrow 0 \text { in probability as } k \rightarrow \infty .
$$

COROLLARY 5. (Theorem of Hsu and Robbins for random elements taking values in Banach space) If $\left\{X_{n}, n \geq 1\right\}$ is a sequence of independent, identically distributed B-valued random elements with $E X_{1}=0$ and $E\left\|X_{1}\right\|^{2}<\infty$, then

$$
\sum_{k=1}^{\infty} P\left[\left\|S_{n_{k}}\right\| \geq n_{k} \varepsilon\right]<\infty .
$$

PROOF. It is easy to see the that conditions ( $\left.\mathrm{I}^{*}\right)$ - (III*) from Corollary 4 are satisfied by the assumptions $E X_{1}=0$ and $E\left\|X_{1}\right\|^{2}<\infty$. Moreover, by the strong law of large numbers for a sequence $\left\{X_{n}, n \geq 1\right\}$ of independent, identically distributed random elements we conclude that

$$
\left\|S_{n} / n\right\| \rightarrow 0 \text { in probability as } n \rightarrow \infty .
$$

COROLLARY 6. Let $\left\{X_{n}, n \geq 1\right\}$ be a sequence of independent, identically distributed B-valued random elements with $E X_{1}=0$ and let $\left\{n_{k}, k \geq 1\right\}$ be a strictly increasing sequence of positive integers. Suppose that for some $r, 1<r \leq 2$,

$$
x^{-r} M(\psi(x)) \rightarrow \infty \text { as } x \rightarrow \infty,
$$


where $\psi(x)=\operatorname{cord}\left\{k: n_{k} \leq x\right\}, r>0, \psi(0)=0, M(x)=\sum_{k=1}^{[x]} n_{k}, x>0$.

If

$$
\sum_{k=1}^{x} n_{k} P\left[\left\|X_{1}\right\| \geq n_{k}\right]<\infty
$$

then

$$
\sum_{k=1}^{\infty} P\left[\left\|S_{n_{k}}\right\| \geq n_{k} \varepsilon\right]<\infty
$$

PROOF. The assumption (3.7) implies that $E M\left(\psi\left(\left\|X_{1}\right\|\right)\right)<\infty$ which with (3.6) gives $E\left\|X_{1}\right\|^{\prime}<\infty$ for some $r, 1<r \leq 2$.

Now it is easy to show that there exists some positive integer $\mathrm{j}$, for which

$$
\begin{gathered}
\sum_{k=1}^{\infty}\left(n_{k}^{-3} E\left\|X_{1}\right\|^{4} I\left[\left\|X_{1}\right\|<2 n_{k} \varepsilon\right]\right)^{2 \jmath} \leq \sum_{k=1}^{\infty}\left(n_{k}^{-3} E\left\|X_{1}\right\|^{r}\left(2 n_{k} \varepsilon\right)^{4-r}\right)^{2} \\
\leq C \cdot \sum_{k=1}^{\infty} n_{k}^{(1-r) 2^{\jmath}}\left(E\left\|X_{1}\right\|^{r}\right)^{2^{\jmath}}<\infty .
\end{gathered}
$$

and

$$
\begin{gathered}
\sum_{k=1}^{\infty}\left(n_{k}^{-1} E\left\|X_{1}\right\|^{2} I\left[\left\|X_{1}\right\|<2 n_{k} \varepsilon\right]\right)^{2 j+1} \leq \sum_{k=1}^{\infty}\left(n_{k}^{-1} E\left\|X_{1}\right\|^{r}\left(2 n_{k} \varepsilon\right)^{2-r}\right)^{2^{j+1}} \\
\leq C^{\prime} \sum_{k=1}^{\infty} n_{k}^{(1-1) 2^{j+1}}\left(E\left\|X_{1}\right\|^{r}\right)^{2^{j+1}}<\infty
\end{gathered}
$$

Similary, as in the proof of Corollary 5 , by the strong law of large numbers for a sequence $\left\{X_{n}, n \geq 1\right\}$ of independent, identically distributed random elements we conclude that

$$
\left\|S_{n_{k}} / n_{k}\right\| \rightarrow 0 \text { in probability as } k \rightarrow \infty
$$

REMARK. Note that the WLLN is implied by the additional conditions: $E X_{n}=0$ and B is of the type 2 since

$$
\begin{gathered}
P\left[\left\|S_{n_{k}}\right\| \geq n_{k} \varepsilon\right] \leq P\left[\left\|S_{n_{k}}-E S_{n_{k}}\right\| \geq n_{k} \varepsilon\right] \\
\leq P\left[\left\|S_{n_{k}}^{\prime}-E S_{n_{k}}^{\prime}\right\| \geq n_{k} \varepsilon\right]+\sum_{i=1}^{n_{k}} P\left[\left\|X_{\imath}\right\| \geq n_{k} \varepsilon /\left(2 \cdot 3^{\jmath}\right)\right] \\
\leq \varepsilon^{-2} n_{k}^{-2} \sum_{i=1}^{n_{k}} E\left\|X_{\imath}^{\prime}\right\|^{2}+\sum_{i=1}^{n_{k}} P\left[\left\|X_{\imath}\right\| \geq n_{k} \varepsilon /\left(2 \cdot 3^{\jmath}\right)\right]=o(1) .
\end{gathered}
$$

Now we are going to present some results on complete convergence for randomly indexed partial sums of independent, non-identically distributed random elements.

THEOREM 3. Let $\left\{X_{n}, n \geq 1\right\}$ be a sequence of independent, B-valued random elements and $\left\{T_{n}, n \geq 1\right\}$ be positive integer valued random variables. Let $\left\{a_{n}, n \geq 1\right\}$ be strictly increasing positive integers and $\left\{\beta_{n}, n \geq 1\right\}$ be positive constants such that $a_{n} \rightarrow \infty$ as $n \rightarrow \infty$, $\lim \sup _{n \rightarrow \infty} \beta_{n}=\beta<1$ and

$$
\sum_{n=1}^{\infty} P\left[\left|T_{n} / a_{n}-N\right| \geq \beta_{n}\right]<\infty,
$$

where $\mathrm{N}$ is a positive random variables such that for some $\mathrm{A}, \mathrm{B}$, where $\beta<A<B<\infty$, $P[A<N<B]=1$. 
If for some positive integer $\mathrm{j}$ and for any given $\varepsilon>0$

$$
\sum_{k=1}^{2} \sum_{i=1}^{\left[u_{k}\left(\beta+\beta_{k}\right)\right]} P\left[\left\|X_{i}\right\| \geq a_{k} \varepsilon(A-\beta) \beta /\left(2 \cdot 3^{j}\right)\right]<\infty
$$

$$
\sum_{h=1}^{\lambda}\left(a_{k}^{-4} \sum_{i=1}^{\left[a_{k}\left(B+\beta_{h}\right)\right]} E\left\|X_{i}\right\|^{4} I\left[\left\|X_{i}\right\|<2 a_{k} \varepsilon(A-\beta)\right]\right)^{2]}<\infty
$$

(c) $\sum_{k=1}^{x}\left(a_{k}^{-4} \sum_{m=2}^{\left[a_{k}\left(B+\beta_{k}\right)\right]} E\left\|X_{m}\right\|^{2} I\left[\left\|X_{m}\right\|<2 a_{k} \varepsilon(A-\beta)\right] \sum_{i=1}^{m-1} E\left\|X_{i}\right\|^{2} I\left[\left\|X_{\imath}\right\|<2 a_{k} \varepsilon(A-\beta)\right]\right)^{2 \jmath}<\infty$, then

$$
\sum_{k=1}^{\infty} P\left[\left\|S_{T_{k}}\right\| \geq T_{k} \varepsilon\right]<\infty
$$

if

$$
\left\|S_{\left[a_{k}\left(B+\beta_{k}\right)\right]} /\left[a_{k}\left(B+\beta_{k}\right)\right]\right\| \rightarrow 0 \text { in probability as } k \rightarrow \infty .
$$

PROOF. Note that

$$
\begin{gathered}
P\left[\left\|\sum_{i=1}^{T_{n}} X_{i}\right\| \geq T_{n} \varepsilon\right] \\
\leq P\left[\left\|\sum_{i=1}^{T_{n}} X_{\imath}\right\| \geq T_{n} \varepsilon,\left|T_{n} / a_{n}-N\right|<\beta_{n}\right]+P\left[\left|T_{n} / a_{n}-N\right| \geq \beta_{n}\right] \\
\leq P\left[\max _{a_{n}\left(A-\beta_{n}\right)<j<a_{n}\left(B+\beta_{n}\right)}\left\|S_{j}\right\| \geq a_{n} \varepsilon(A-\beta)\right]+P\left[\left|T_{n} / a_{n}-N\right| \geq \beta_{n}\right]
\end{gathered}
$$

Now assuming that $X_{n}, n \geq 1$, are symmetrically distributed random elements we get by the Lévy's inequality

$$
\begin{aligned}
& \left.P \sum_{a_{n}\left(A-\beta_{n}\right)<j<a_{n}\left(B+\beta_{n}\right)}\left\|S_{\jmath}\right\| \geq a_{n} \varepsilon(A-\beta)\right] \\
& \leq 2 P\left[\left\|\sum_{i=1}^{\left[a_{n}\left(B+\beta_{n}\right)\right]} X_{i}\right\| \geq a_{n} \varepsilon(A-\beta)\right] .
\end{aligned}
$$

But under the assumptions of Theorem 3 one can verify after using Theorem 1 with $n_{k}=\left[a_{k}\left(B+\beta_{k}\right)\right]$ that

$$
\sum_{k=1}^{\infty} P\left[\left\|\sum_{i=1}^{\left[a_{k}\left(B+\beta_{k}\right)\right]} X_{\imath}\right\| \geq a_{k} \varepsilon(A-\beta)\right]<\infty
$$

This bound and the assumption (3.8) together with (3.10) imply (3.9) for symmetrically distributed random elements.

To remove the symmetry assumption we proceed similar as it has been done in the proof of Theorem 2.

$$
\sum_{n=1}^{\infty} P\left[\left\|S_{T_{n}} / T_{n}-\operatorname{med}\left(S_{T_{n}} / T_{n}\right)\right\| \geq \varepsilon\right]<\infty .
$$

Now we note that

$$
P\left[\left\|\sum_{\imath=1}^{T_{n}} X_{\imath}\right\| \geq T_{n} \varepsilon\right]
$$




$$
\begin{aligned}
& \leq P\left[\ln _{n\left(1+1-s_{n}\right)<1<i_{n}\left(H+3_{n}\right)}\left\|\sum_{i=1}^{J} X_{i} I\left[\left\|X_{i}\right\|<a_{n}(A-\beta) \varepsilon\right]\right\| \geq a_{n} \varepsilon(A-\beta)\right] \\
& +\sum_{i=1}^{\left[{ }_{n}\left(B+, s_{h}\right)\right]} P\left[\left\|\cdot X_{i}\right\| \geq a_{h}=(A-3)\right]+P\left[\left|T_{n} / a_{n}-V\right| \geq \beta_{n}\right]
\end{aligned}
$$

But by the Kolmogoror's inequality

$$
\begin{aligned}
& P\left[\max _{a_{n}\left(A-\beta_{n}\right)<j<a_{n}\left(B+\beta_{n}\right)}\left\|\sum_{i=1}^{J} X_{i} I\left[\left\|X_{i}\right\|<a_{n}(A-3) \varepsilon\right]\right\| \geq a_{n} \varepsilon(A-\beta)\right] \\
& \quad \leq(\varepsilon(A-\beta))^{-2} a_{n}^{-2} \sum_{i=1}^{\left[n_{n}\left(B+, 3_{n}\right)\right]} E\left\|X_{i}\right\|^{2} I\left[\left\|X_{i}\right\|<a_{n} \varepsilon(A-\beta)\right] .
\end{aligned}
$$

Taking into account that

$$
a_{n}^{-2} \sum_{i=1}^{\left[a_{n}\left(B+\beta_{n}\right)\right]} E\left\|X_{i}\right\|^{2} I\left[\left\|X_{\imath}\right\|<a_{n} \varepsilon(A-\beta)\right] \rightarrow 0 \text { as } n \rightarrow \infty
$$

(cf. the proof of Theorem 1), (3.8) and assumption (a) we have

$$
P\left[\left\|\sum_{i=1}^{T_{n}} X_{i}\right\| \geq T_{n} \Xi\right] \rightarrow 0 \text { as } n \rightarrow \infty .
$$

Therefore, (3.11) and (3.12) imply that

$$
\left\|\operatorname{med}\left(S_{T_{n}} / T_{n}\right)\right\| \rightarrow 0 \text { as } n \rightarrow \infty,
$$

and complete the proof of the Theorem 3 .

Note that Theorem 3 generalizes the results presented by Adler [15].

The following corollary is an extension of Adler's result to independent non-identically distributed B-valued random elements.

COROLLARY 7. Let $\left\{X_{n}, n \geq 1\right\}$ be a sequence of independent, B-valued random elements and $\left\{T_{n}, n \geq 1\right\}$ be positive integer valued random variables. Suppose that $\left\{a_{n}, n \geq 1\right\}$ is a strictly increasing sequence of positive integers and $\left\{\beta_{n}, n \geq 1\right\}$ is a sequence of positive constants such that $a_{n} \rightarrow \infty$ as $n \rightarrow \infty$, lim $\sup _{n \rightarrow \infty} \beta_{n}=\beta<1$ and

$$
\sum_{n=1}^{\infty} P\left[\left|T_{n} / a_{n}-1\right| \geq \beta_{n}\right]<\infty
$$

If for some positive integer $\mathrm{j}$ and for any given $\varepsilon>0$ the assumptions $(a)-(c)$ are satisfied then

$$
\sum_{k=1}^{\infty} P\left[\left\|S_{T_{k}}\right\| \geq T_{k} \varepsilon\right]<\infty
$$

if

$$
\left\|S_{\left[a_{k}\left(1+\beta_{k}\right)\right]} /\left[a_{k}\left(1+\beta_{k}\right)\right]\right\| \rightarrow 0 \text { in probability as } k \rightarrow \infty .
$$

The next corollary is an extension of one of the results given in Adler [15] to the case of i.i.d. B-valued random elements. 
COROLIARY 8 . Let $\left\{X_{n}, n \geq 1\right\}$ be a sequence of independent identically distributed B-valued random elements with $E X_{1}=0$ and $\left\{T_{n}, n \geq 1\right\}$ be a sequence of positive integervalued random variables. Suppose that $\left\{a_{n}, n \geq 1\right\}$ is a strictly increasing sequence of positive integers and $\left\{\beta_{n}, n \geq 1\right\}$ is a sequence of positive constants such that $a_{n} \rightarrow \infty$ as $n \rightarrow \infty$, $\operatorname{lom} s u p_{n-x} \beta_{n}=\beta<1$ and

$$
\sum_{n=1}^{\infty} P\left[\left|T_{n} / a_{n}-1\right| \geq \beta_{n}\right]<\infty
$$

Suppose that for some $r, 1<r \leq 2, x^{-r} M(\psi(x)) \rightarrow \infty$ as $x \rightarrow \infty$, where $\psi(x)=\operatorname{card}\left\{k: a_{\curlywedge} \leq x\right\}, x>0, \psi(0)=0, M(x)=\sum_{k=1}^{[x]} a_{k}, x>0$. If $\sum_{k=1}^{\infty} a_{k} P\left[\left\|X_{1}\right\| \geq a_{k} \varepsilon\right]<\infty$ then

$$
\sum_{k=1}^{\infty} P\left[\left\|S_{T_{k}}\right\| \geq T_{k} \varepsilon\right]<\infty .
$$

ACKNOWLEDGEMENT. We are very grateful to the referee for his helpful comments allowing us to improve the previous version of the paper.

\section{REFERENCES}

1. HSU, P.L. and ROBBINS, H. Complete Convergence and the Law of Large Numbers. Proc. Nat. Acad. Sci. U.S.A. 33 (1947) 25- 31.

2. ERDÖS, P. On a Theorem of Hsu-Robbins.Ann. of Math. Statist. 20 (1949) 286-291.

3. KATZ, M. The Probability in the Tail of Distribution. Ann. Math. Statist. 34 (1963) 312-318.

4. BAUM, L.E. and KATZ, M. Convergence Rates in the Law of Large Numbers. Trans. Amer. Math. Soc., 120 (1965) 108-123.

5. ASMUSSEN, S. and KURTZ, T. Necessary and Sufficient Conditions for Complete Convergence in the Law of Large Numbers.Ann. Probab., 8 (1980) 176-182.

6. GUT, A. On Complete Convergence in the law of Large Numbers for Subsequences. Ann. Probab. 13 (1985) 1286-1291.

7. DUNCAN, R. and SZYNAL, D. A Note on the Weak and Hsu-Robbins Law of Large Numbers. Bull. Ac. Pol. Math., 32 (1984) 729-735.

8. BARTOSZYNSKI, R. and PURI, P.S. On the Rate of Convergence for the Weak Law of Large Numbers. Probab. Math. Statist., 5 (1985) 91-97.

9. KUCZMASZEWSKA, A. and SZYNAL, D. On the Law of Large Numbers of the Hsu-Robbins Type. Probab. Math. Statist. 9 (1988) 85-93.

10. KUCZMASZEWSKA, A. and SZYNAL, D. On the Hsu-Robbins Law of Large Numbers for Subsequences. Bull. Ac. Pol. Math. 36 (1988) 69-79.

11. HOFFMANN-JÖRGENSEN, J. Sums of Independent Banach Spaces Valued Random Variables. Studia Math. 50 (1974) 159-186.

12. YURINSKI, V.V. Exponental Bounds for Large Deviations. Theor. Prob. Appl. 19 (1974) 154-155.

13. LOĖVE, M. Probability Theory. 4rd ed. Vol 1 Van Nostrand Princeton, (1977).

14. TAYLOR, R. Stochastic Convergence of Weighted Sums of Random Elements in Linear Space. Lecture Notes in Mathematics. 672 Springer- Verlag, Berlin, (1978).

15. ADLER, A. On Complete Convergence of the Sum of a Random Number of Random Variables. Calcutta Statist. Assoc. Bull., 37 (1988) 161-169. 


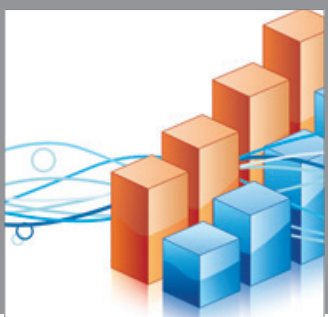

Advances in

Operations Research

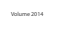

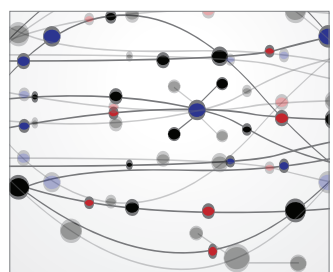

\section{The Scientific} World Journal
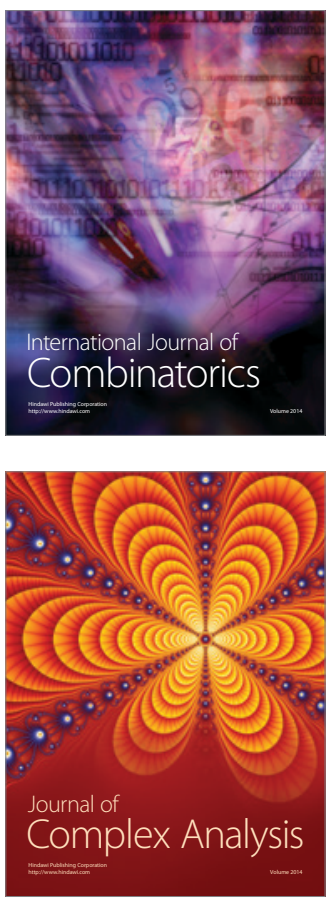

International Journal of

Mathematics and

Mathematical

Sciences
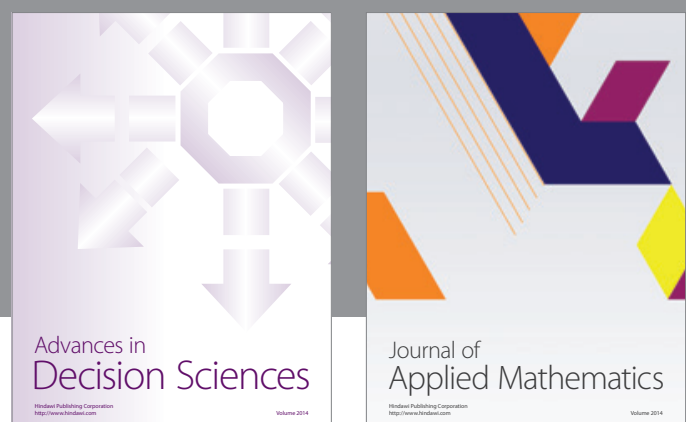

Journal of

Applied Mathematics
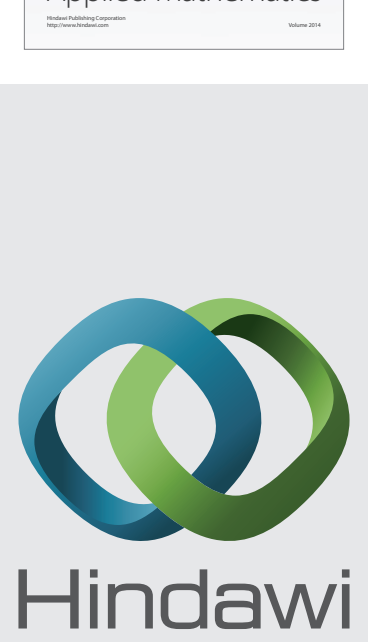

Submit your manuscripts at http://www.hindawi.com
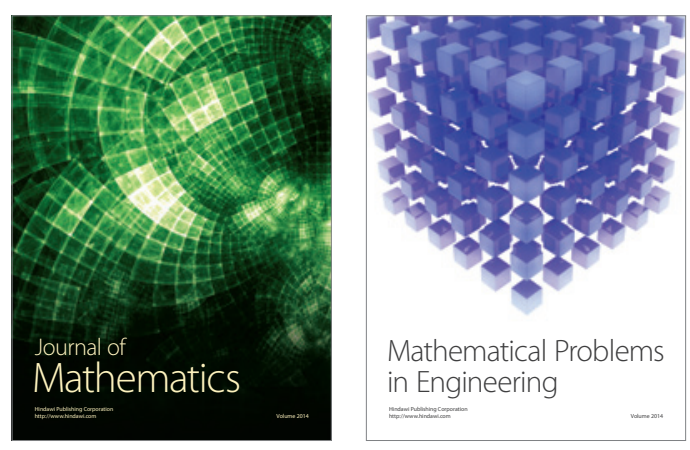

Mathematical Problems in Engineering
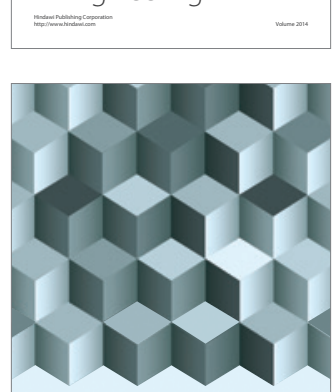

Journal of

Function Spaces
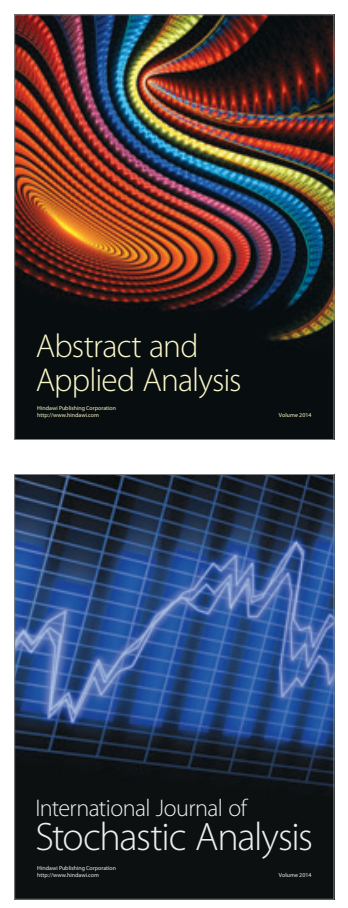

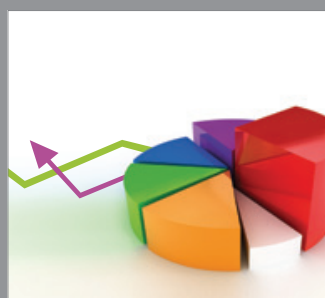

ournal of

Probability and Statistics

Promensencen
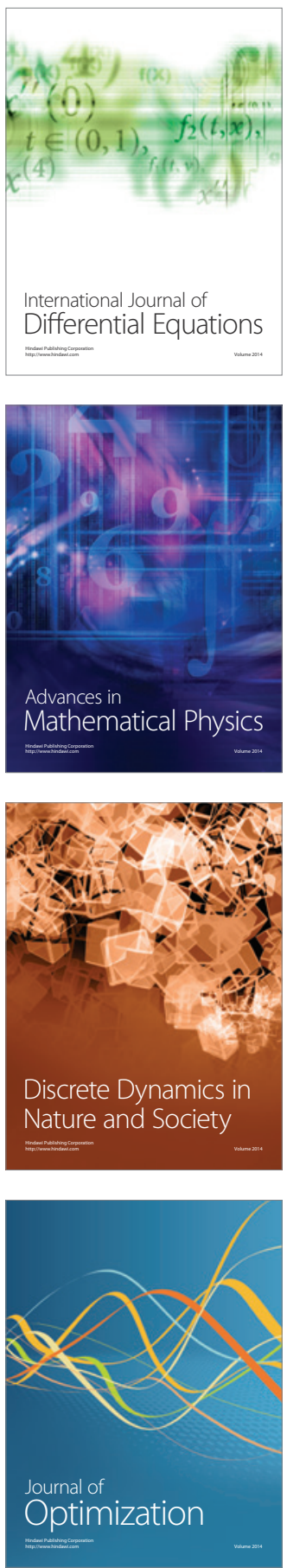